\title{
NODE-DISJOINT CYCLES IN BRIDGELESS CUBIC GRAPHS
}

\author{
THINH D. NGUYEN
}

\begin{abstract}
We prove the NP-hardness of deciding whether a connected bridgeless cubic graph $G(V, E)$ contains two node-disjoint cycles of equal cardinality $|V| / 2$.
\end{abstract}

Definition 1. (Two Disjoint Cycles problem): As stated in the abstract.

Definition 2. (Ham-Cycle Avoiding Edge): Does a 2-edge-connected cubic graph $H$ contain a Hamiltonian cycle avoiding a given edge $e$

Claim 3. We have that Ham-Cycle Avoiding Edge $\leq_{p}$ Two Disjoint Cycles

Proof. Construct $G$ as follows. Take two copies of $H-e$, denoting the endpoints of $e$ in the first copy $v_{1}, u_{1}$ and denoting the endpoints of $e$ in the second copy $v_{2}$, $u_{2}$. Add edges $e_{v}$ between $v_{1}$ and $v_{2}$, and $e_{u}$ between $u_{1}$ and $u_{2}$. Observe that $G$ is 2-edge-connected.

Any cycle of $G$ contains both or neither of $e_{v}$ and $e_{u}$, since these edges form an edge cut. Thus if $G$ has two vertex-disjoint cycles of length $|V(H)|$, they must each lie in a separate copy of $H$. Such cycles exist precisely if $H$ contains a Hamiltonian cycle avoiding $e$.

\section{CONCLUSION}

As long as we study a mathematical conjecture, we should encourage ourselves of having enough labouring hours on popular maths books. Then, reading some articles on theory of computing like [3] is a good practice. Only after that, could we think of the ultimate final for all mathematics sciences.

\section{REFERENCES}

1. Michael R. Garey, David S. Johnson, Computers and Intractability: A Guide to the Theory of NP-Completeness

2. David S. Johnson, The NP-Completeness Column: An Ongoing Guide. pp.393-405

3. Phan Dinh Dieu, Le Cong Thanh, Le Tuan Hoa, Average Polyno-mial Time Complexity of Some NP-Complete Problems, Theor. Comput. Sci. 46(3): pp.219-237 (1986)

Current address: Department of Mathematics, Moscow State University

Email address: kosmofarmer@yandex.com

Key words and phrases. balanced, bridgeless, cycle, cubic, disjoint, graph. Perebor. 